\title{
Wave reflection and transmission by curtainwall-pile breakwaters using circular piles
}

\author{
Kyung-Duck Suh ${ }^{\mathrm{a}, *}$, Hoo Young Jung ${ }^{\mathrm{b}}$, Chong Kun Pyun ${ }^{\mathrm{c}}$ \\ ${ }^{a}$ Department of Civil, Urban, and Geosystem Engineering \& Engineering Research \\ Institute, Seoul National University, San 56-1, Shillim-dong, Gwanak-gu, Seoul 151-744, \\ South Korea \\ ${ }^{\mathrm{b}}$ Harbor Division, Seil Engineering Co., Ltd., Jung Ang Building, 65-106, Shingil-dong, \\ Youngdungpo-gu, Seoul 150-051, South Korea \\ ${ }^{c}$ Department of Civil and Environmental Engineering, Myongji University, San 38-2, \\ Nam-dong, Cheoin-gu, Yongin, Gyeonggi-do 449-728, South Korea
}

\begin{abstract}
This paper presents a mathematical model which computes the hydrodynamic characteristics of a curtainwall-pile breakwater using circular piles, by modifying the model developed for rectangular piles by Suh et al. in 2006. To examine the validity of the model, laboratory experiments have been conducted for curtainwall-pile breakwaters with various values of draft of curtain wall, spacing between piles, and wave height and period. Comparisons between measurement and prediction show that the mathematical model adequately reproduces most of the important features of the experimental results. The mathematical model based on linear wave theory tends to over-predict the reflection coefficient as the wave height increases. As the draft of the curtain wall increases and the porosity between piles decreases, the reflection and transmission coefficient increases and decreases, respectively, as expected. As the relative water depth increases, however, the effect of porosity disappears because the wave motion is minimal in the lower part of a water column for short waves.
\end{abstract}

Keywords: Breakwaters; Curtain walls; Laboratory tests; Mathematical models; Piles; Wave reflection; Wave transmission.

\footnotetext{
* Corresponding author. Tel.: +82 288087 60; fax: +82 288703 49. Email addresses: kdsuh@snu.ac.kr (K.-D. Suh), punkshow@ mju.ac.kr (H.Y. Jung), ckpyun@ mju.ac.kr (C.K. Pyun)
} 


\section{Introduction}

In general, the width of gravity-type breakwaters increases with water depth, leaving a large footprint and requiring a great amount of construction material, especially when built in deeper water. Often they block littoral drift and cause severe erosion or accretion in neighboring beaches. In addition, they prevent the circulation of water and so deteriorate the water quality within the harbor. In some places, they obstruct the passage of fishes and bottom dwelling organisms. A solid soil foundation is also needed to support such heavy structures.

In order to resolve the above-mentioned problems, porous (permeable) structures have been introduced especially in small craft harbors. The simplest porous structure may be a curtain wall breakwater, which consists of a vertical wall extending from the water surface to some distance above the sea bed, or a pile breakwater, which consists of an array of closely spaced vertical piles. Recently, Suh et al. (2006) proposed a curtainwall-pile breakwater (CPB hereinafter), the upper part of which is a vertical wall and the lower part consists of an array of vertical piles. They developed a mathematical model to predict wave transmission, reflection, run-up, and wave force acting on a CPB, using the eigenfunction expansion method. They conducted large-scale laboratory experiments to examine the validity of the developed model, showing that the model adequately reproduces most of the important features of the experimental results.

A CPB is being constructed at the Yeoho Port in the south coast of Korea, the crosssection of which is shown in Fig. 1. The vertical pile indicates the CPB, and the right inclined pile is constructed more sparsely than the vertical pile to support the breakwater. The curtain wall is installed in front of the vertical piles and is extended to $1.1 \mathrm{~m}$ below the datum level. The sea bed is located at $1.6 \mathrm{~m}$ below the datum level so that only $0.5 \mathrm{~m}$ above the sea bed is open. The trapezoidal concrete pedestals between 1.1 and $1.6 \mathrm{~m}$ below the datum level (see Fig. 1) are attached to the piles and support the curtain wall so that an opening is formed between adjacent piles. The CPB of the Yeoho Port uses circular piles, and the thickness of the curtain wall is smaller than the pile diameter. Suh et al. (2006) used square piles, the side length of which is the same as the thickness of the curtain wall. Also they tested CPB's only for one spacing between piles. In this paper, we modify Suh et al.'s (2006) model to be used for circular piles. To examine the validity of the model, laboratory experiments are conducted for CPB's with various values of 
draft of curtain wall, spacing between piles, and wave height and period. In the following section, the modification of the mathematical model of Suh et al. (2006) for circular piles is described. In Section 3, the laboratory experiment is described. In Section 4, the predictions of the model are compared with the experimental results. The major conclusions then follow.

\section{Mathematical model}

Let us consider the CPB sketched in Fig. 2, in which $h$ is constant water depth in still water, $d$ draft of the curtain wall, $b$ thickness of the wall, and $D$ is the diameter of the pile. This CPB is different from the Yeoho Port breakwater in that the curtain wall is not located in front of the piles but located between the piles. This difference, however, may not significantly change the hydrodynamic characteristics of the breakwater. A Cartesian coordinate system $(x, z)$ is defined with the positive $x$ directing downwave from the crest line of the breakwater and the vertical coordinate $z$ being measured vertically upwards from the still water line. The distance between the centers of two neighboring piles is denoted as $2 A$ and the width of an opening is $2 a$ so that the porosity of the lower part of the breakwater at $x=0$ is defined as $r_{0}=a / A$. A regular wave train with wave height $H_{i}$ is incident in the positive $x$-direction. We divide the fluid domain into region $1(x \leq 0)$ and region $2(x \geq 0)$.

Assuming incompressible fluid and irrotational flow motion, the velocity potential $\Phi$ exists, which satisfies the Laplace equation. In addition, the wave height is assumed sufficiently small so that $\Phi$ is subjected to the linearized free-surface boundary condition. Then the velocity potential $\Phi(x, z, t)$ in water of constant depth $h$ [see, for example, Dean and Dalrymple (1991)] may be expressed, using complex notation, as

$$
\Phi(x, z, t)=\operatorname{Re}\left\{-\frac{i g H_{i}}{2 \omega} \frac{1}{\cos \operatorname{lk}(h)} \phi(x, z) \exp -(i \omega t)\right\}
$$

where $\omega$ is the wave angular frequency, $g$ the gravitational acceleration, $i=\sqrt{-1}$, and the symbol Re represents the real part of a complex value. The wave number $k$ must satisfy the dispersion relationship: 


$$
\omega^{2}=g k \mathrm{tan} \mathrm{kk}(h)
$$

The spatial variation of the velocity potential $\phi(x, z)$ should be determined in each region.

We assume that the wall thickness is very small compared with the wave length, so that the wall has no thickness mathematically. Then $\phi_{1}(x, z)$ and $\phi_{2}(x, z)$ must satisfy the following matching conditions at $x=0$ :

$$
\begin{aligned}
& \frac{\partial \phi_{1}}{\partial x}=\frac{\partial \phi_{2}}{\partial x}=0 \quad \text { for }-d \leq z \leq 0, \quad x=0 \\
& \frac{\partial \phi_{1}}{\partial x}=\frac{\partial \phi_{2}}{\partial x}=i G\left(\phi_{1}-\phi_{2}\right) \quad \text { for }-h \leq z \leq-d, \quad x=0
\end{aligned}
$$

where the subscripts indicate the regions of the fluid domain. The first matching condition describes that the horizontal velocities vanish on both sides of the upper impermeable wall of the breakwater. The second one for the lower part of the breakwater describes that the horizontal velocities in the two regions must be same at the breakwater and that the horizontal velocity at the opening is proportional to the difference of velocity potentials, or the pressure difference, across the breakwater. The proportional constant $G$, often called permeability parameter, is in general complex. There are several ways to express the constant $G$. In the present study, we adopt the method of Mei et al. (1974) and $G$ is expressed by

$$
G=\frac{1}{\frac{\beta}{\omega}-i \ell}
$$

where $\beta$ is the energy dissipation coefficient derived by linearizing the nonlinear convective acceleration term in the equation of motion, and $\ell$ is the length of the jet flowing through the gap between piles. The real part of the denominator in (5) corresponds to the resistance of the breakwater and the imaginary part is associated with the phase difference between the velocity and the pressure due to inertial effects. 
The linearized dissipation coefficient $\beta$ is given by Kim (1998) as

$$
\beta=\frac{8 \alpha}{9 \pi} H_{i} \omega \frac{1}{\sqrt{(R+2)^{2}+P^{2}}} \frac{5+\mathrm{cos} \mathrm{Q} k h)}{2 k h+\mathrm{sinh} 2(k h)}
$$

where $P=\ell k, R=\beta k / \omega$, and $\alpha$ is the head loss coefficient. The preceding equation was derived for a pile breakwater without a curtain wall, but it could be used for a CPB because the mechanism of energy dissipation between piles must be same for these two breakwaters. Rearrangement of (6) gives a quartic polynomial of $\beta$, which can be solved by the eigenvalue method [e.g., Press et al. (1992)].

Suh et al. (2002) showed that the jet length $\ell$ is related to the blockage coefficient $C$ by

$$
\ell=2 C
$$

Kakuno and Liu (1993) proposed the blockage coefficient for circular piles as

$$
C=\frac{\pi}{4} A\left(1-r_{0}\right)^{2} \frac{1}{1-\xi} ; \quad \xi=\frac{\pi^{2}}{12}\left(1-r_{0}\right)^{2}
$$

Park et al. (2000) proposed a formula for the head loss coefficient:

$$
\alpha=\left(\frac{1}{\bar{r} C_{c}}-1\right)^{2}
$$

where the ad hoc porosity $\bar{r}$ is given by

$$
\frac{1}{\bar{r}^{2}}=\frac{1}{D} \int_{-D / 2}^{D / 2} \frac{d x}{r(x)^{2}}
$$

with the spatially varying porosity 


$$
r(x)=1-\frac{\sqrt{\left(\frac{D}{2}\right)^{2}-x^{2}}}{\frac{D}{2}+a}
$$

and $C_{c}$ is the empirical contraction coefficient, for which Mei et al. (1974) suggested using the formula:

$$
C_{c}=0.6+0.4 r_{0}^{2}
$$

To obtain the solutions for $\phi_{1}$ and $\phi_{2}$, we use the eigenfunction expansion method. We closely follow the method of Isaacson et al. (1998), which was also used by Suh et al. (2006). The velocity potential is expressed in a series of infinite number of solutions:

$$
\begin{aligned}
& \left.\phi_{1}=\phi_{i}-\sum_{m=0}^{\infty} A_{m} \cos \left[\mu_{m}(h+z)\right] \mathrm{exp} \mu_{m} x\right) \\
& \phi_{2}=\phi_{i}+\sum_{m=0}^{\infty} A_{m} \cos \left[\mu_{m}(h+z)\right] \mathrm{e} \times \mathrm{p}-\left(\mu_{m} x\right)
\end{aligned}
$$

where $\phi_{i}=\cosh [k(h+z)] \exp (i k x)$ is the incident wave potential. The wave numbers $\mu_{m}$ are the solution of the dispersion relation, $\omega^{2}=-g \mu_{m} \tan \left(\mu_{m} h\right)$, which has an infinite discrete set of real roots $\pm \mu_{m}(m \geq 1)$ for non-propagating waves and a pair of imaginary roots $\mu_{0}= \pm i k$ for propagating waves. We take $\mu_{0}=-i k$ so that the propagating waves in (13) and (14) correspond to reflected and transmitted waves, respectively. We also take the positive roots for $m \geq 1$ so that the non-propagating waves die out exponentially with the distance from the breakwater.

Now the solutions (13) and (14) satisfy the free surface boundary condition and the bottom boundary condition. Also, they automatically satisfy the requirement that the horizontal velocities must be matched at the breakwater. In order to solve for the 
unknown coefficients $A_{m}$ 's, we use the matching conditions at the breakwater. First, (13) and (14) are substituted into (3) and (4), respectively. Multiplying each resulting equation by $\cos \left[\mu_{n}(h+z)\right]$, integrating with respect to $z$ over the appropriate domain of $z$ (i.e., $z=-d$ to 0 , or $z=-h$ to $-d$ ), and finally adding them, we obtain a matrix equation for $A_{m}$ :

$$
\sum_{m=0}^{\infty} C_{m n} A_{m}=b_{n} \quad \text { for } n=0,1,2, \cdots, \infty
$$

where

$$
\begin{aligned}
& C_{m n}=\mu_{m} f_{m n}(-d, 0)+\left(\mu_{m}-2 i G\right) f_{m n}(-h,-d) \\
& b_{n}=-\mu_{0}\left[f_{0 n}(-d, 0)+f_{0 n}(-h,-d)\right] \\
& f_{m n}(p, q)=\int_{p}^{q} \cos \left[\mu_{m}(h+z)\right] \cos \left[\mu_{n}(h+z)\right] d z \\
& \quad= \begin{cases}\frac{1}{2}\left[\frac{\sin \left[\left(\mu_{m}+\mu_{n}\right)(h+z)\right]}{\mu_{m}+\mu_{n}}+\frac{\sin \left[\left(\mu_{m}-\mu_{n}\right)(h+z)\right]}{\mu_{m}-\mu_{n}}\right]_{p}^{q} & \text { for } m \neq n \\
\frac{1}{4 \mu_{\mathrm{m}}}\left[2 \mu_{m}(h+z)+\sin \left[2 \mu_{m}(h+z)\right]\right]_{p}^{q} & \text { for } m=n\end{cases}
\end{aligned}
$$

Once the wave potentials are calculated, we can obtain various engineering wave properties. The reflection and transmission coefficients are given by

$$
C_{r}=\left|A_{0}\right|
$$

and

$$
C_{t}=\left|1+A_{0}\right|
$$

respectively. The wave run-up on the upwave face of the breakwater is given by 


$$
\left.R_{u}=\frac{H_{i}}{2} \mid 1-\frac{1}{\cos 1 k(h)} \sum_{m=0}^{\infty} A_{m} \cos \mu_{m} h\right) \mid
$$

Since the vertical distributions of wave pressure on both upwave and downwave sides of the breakwater are known, the wave force and overturning moment about the mud line can also be calculated. The maximum horizontal wave force $F_{\max }$ and the maximum overturning moment about the mud line $M_{\max }$ per unit width of the breakwater are given by

$$
F_{\text {m a }}=\rho g H_{i} \frac{1}{\cosh (k h)}\left|\sum_{m=0}^{\infty} \frac{A_{m}}{\mu_{m}}\left\{\sin \left(\mu_{m} h\right)-r_{0} \sin \left[\mu_{m}(h-d)\right]\right\}\right|
$$

and

$$
\begin{aligned}
M_{\mathrm{max}}=\rho g H_{i} \frac{1}{\cosh (k h)} \mid \sum_{m=0}^{\infty} \frac{A_{m}}{\mu_{m}^{2}}\left\{\cos \left(\mu_{m} h\right)+\mu_{m} h \sin \left(\mu_{m} h\right)\right. \\
\left.\quad-r_{0} \cos \left[\mu_{m}(h-d)\right]-r_{0} \mu_{m}(h-d) \sin \left[\mu_{m}(h-d)\right]-1+r_{0}\right\} \mid
\end{aligned}
$$

respectively, where $\rho$ is the density of fluid.

\section{Laboratory experiment}

Experiments were carried out in the wave flume at Department of Civil and Environmental Engineering of Myongji University. The flume was $30 \mathrm{~m}$ long, $0.7 \mathrm{~m}$ wide, and $1.4 \mathrm{~m}$ deep. It was equipped with a piston-type wave generator at one end, and a wave absorbing beach at the other. A mortar-covered false bed with a 1/10 fore-slope of $2.5 \mathrm{~m}$ length followed by a $1 / 30$ slope of $6 \mathrm{~m}$ length was constructed at the elevation of $0.45 \mathrm{~m}$ from the bottom of the flume. The fore-slope of the false bed started at a distance of $6 \mathrm{~m}$ from the wave maker. The breakwater model was placed at a distance of $22.5 \mathrm{~m}$ from the wave maker and $8 \mathrm{~m}$ from the beginning of the flat false bed. Steel pipes and 
acrylic plates were used to make the piles and curtain walls, respectively. Water surface displacements were measured with capacitance-type wave gauges.

All experiments were conducted at a water depth of $0.37 \mathrm{~m}$ on the false bed. Circular piles of $7 \mathrm{~cm}$ diameter were used with $a=1.5,2.33,3.5$, and $5.25 \mathrm{~cm}$, which corresponds to $7,6,5$, and 4 piles, respectively, in the flume of $0.7 \mathrm{~m}$ width. The corresponding porosity of the lower perforated wall was $0.3,0.4,0.5$, and 0.6. The thickness of the curtain wall $b$ was $3.5 \mathrm{~cm}$. Five different drafts of the curtain wall were used; $12,14,16.8,21.1$, and $28.1 \mathrm{~cm}$. The curtain wall was high enough above the water level to prevent wave overtopping.

Three different wave periods ( $T=0.8,1.08,1.5 \mathrm{~s}$ ) were used except for the cases of porosity of 0.5 , in which additional tests were made for $1.3 \mathrm{~s}$ wave period. For each of the wave period, three different wave heights were tested; 7.2, 9.2, and $11.2 \mathrm{~cm}$. The relative depth $k h$ ranges from 0.91 to 2.4 , and the wave steepness $H / L$ ranges between 0.0283 and 0.114 .

To measure the incident and reflected waves, the spatial variation of the wave envelope was measured by slowly moving a wave gauge in the uniform depth region in front of the breakwater (see Dean and Dalrymple, 1991). The transmitted waves were measured using a wave gage behind the breakwater assuming that the wave reflection from the downwave beach is negligible. Previous observations indicated reflection coefficients from the beach of 0.05 to 0.1 for the wave periods used in these tests. Wave measurements were made for $60 \mathrm{~s}$ at a sampling rate of $20 \mathrm{~Hz}$ immediately after the initiation of wave generation. For the analysis of wave reflection and transmission, we used 512 data after skipping the first $20 \mathrm{~s}$.

\section{Comparison with experimental results}

In this section, the mathematical model results are compared with the experimental results. The number of terms used in the eigenfunction expansion method was 50, which was found to give accurate results over the range of values presented here.

Comparison of the measured and calculated reflection coefficients is shown in Fig. 3 for different wave heights, in which the subscripts $\mathrm{c}$ and $\mathrm{m}$ denote calculation and measurement, respectively. Although the overall agreement is acceptable, the model somewhat over-predicts the reflection coefficients at larger values, while under- 
predicting them at smaller values. The over-prediction at larger values is more apparent for larger wave heights, for which the linear wave theory used in this study becomes inaccurate. Fig. 4 shows a comparison of the measured and calculated transmission coefficients. Again the overall agreement is acceptable, but the model somewhat overpredicts the transmission coefficient except at very large or very small values. In this case, there is no evidence of the effect of wave nonlinearity.

In order to show the effect of the draft of the curtain wall, a selection of results is presented in Figs. 5 and 6, which show comparisons of the measured and predicted reflection and transmission coefficients, each as a function of $k h$ for porosity between piles $r_{0}=0.4$ and 0.6, respectively. Comparisons are shown for relative drafts $d / h=0.324,0.570$, and 0.759 . For the experimental data, those of the smallest wave height $H=7.2 \mathrm{~cm}$ were used, which would give good agreement with the linear wave theory. In general, the mathematical model adequately reproduces most of the important features of the experimental results. The reflection and transmission coefficients, respectively, increase and decrease with the relative water depth. As the draft of the curtain wall increases, the reflection coefficient increases while the transmission coefficient decreases, as expected, in both prediction and measurement. A comparison between Figs. 5 and 6 shows that the reflection and transmission coefficient decreases and increases, respectively, with the porosity between piles, especially for smaller relative water depth.

Figs. 7 and 8 show the effect of the porosity between piles, which compares the measured and predicted reflection and transmission coefficients, as a function of $k h$ for relative drafts $d / h=0.378$ and 0.570 , respectively. Comparisons are shown for porosities between piles $r_{0}=0.3,0.4,0.5$, and 0.6. Again the experimental data of the smallest wave height $H=7.2 \mathrm{~cm}$ were used. As the porosity between piles increases, the reflection coefficient decreases while the transmission coefficient increases, as expected, in both prediction and measurement. As the relative water depth increases, however, the effect of porosity disappears because the wave motion is minimal in the lower part of the water column for short waves. A comparison between Figs. 7 and 8 shows that the reflection and transmission coefficient increases and decreases, respectively, with the relative draft of curtain wall.

\section{Conclusions}


In this study, we modified the mathematical model of Suh et al. (2006), which was developed for a $\mathrm{CPB}$ using rectangular piles, to be used for circular piles. The model was then compared with the experimental data obtained for various values of draft of curtain wall, spacing between piles, and wave height and period.

Comparisons between measurement and prediction showed that the mathematical model was able to adequately reproduce most of the important features of the experimental results, even though the reflection coefficients were over-predicted for larger wave heights, which violate the linear wave theory. The reflection coefficient increased with the relative water depth, whereas the transmission coefficient decreased with the relative depth. As the draft of the curtain wall increased, the reflection coefficient increased, while the transmission coefficient decreased, as expected. On the other hand, as the porosity between piles increased, the reflection coefficient decreased while the transmission coefficient increased. As the relative water depth increased, however, the effect of porosity disappeared because the wave motion was minimal in the lower part of the water column for short waves.

\section{Acknowledgements}

KDS was supported by the Korea Sea Grant Program and the Brain Korea 21 Project.

\section{References}

Dean, R.G., Dalrymple, R.A., 1991. Water Wave Mechanics for Engineers and Scientists. World Scientific, Singapore, 353 pp.

Isaacson, M., Premasiri, S., Yang, G., 1998. Wave interactions with vertical slotted barrier. Journal of Waterway, Port, Coastal and Ocean Engineering 124(3), 118-126.

Kakuno, S., Liu, P.L.-F., 1993. Scattering of water waves by vertical cylinders. Journal of Waterway, Port, Coastal and Ocean Engineering 119(4), 302-322.

Kim, B.H., 1998. Interactions of Waves, Seabed and Structures. PhD Thesis, Seoul National University, 246 pp.

Mei, C.C., Liu, P.L.-F., Ippen, A.T., 1974. Quadratic loss and scattering of long waves. Journal of Waterway, Harbors, and Coastal Engineering Division, ASCE 100(3), 217- 
239.

Park, W.S., Kim, B.H., Suh, K.D., Lee, K.S., 2000. Scattering of irregular waves by vertical cylinders. Coastal Engineering Journal 42(2), 253-271.

Press, W.H., Teukolsky, S.A., Vetterling, W.T., Flannery, B.P., 1992. Numerical Recipes in FORTRAN: the Art of Scientific Computing. Cambridge University Press, Cambridge, 963 pp.

Suh, K.-D., Shin, S., Cox, D.T., 2006. Hydrodynamic characteristics of pile-supported vertical wall breakwaters. Journal of Waterway, Port, Coastal and Ocean Engineering 132(2), 83-96.

Suh, K.D., Son, S.Y., Lee, J.I., Lee, T.H., 2002. Calculation of irregular wave reflection from perforated-wall caisson breakwaters using a regular wave model. Proceedings of the 28th International Conference on Coastal Engineering, vol. 2. ASCE, Cardiff, UK, pp. 1709-1721. 


\section{Caption of figures}

1. Cross-section of the Yeoho Port breakwater.

2. Definition sketch: (a) side view, (b) front view and top view.

3. Comparison of the reflection coefficients between measurement and calculation.

4. Comparison of the transmission coefficients between measurement and calculation.

5. Comparison of the predicted reflection and transmission coefficients with experimental results as a function of $k h$ for $r_{0}=0.4$ : (a) reflection coefficient, (b) transmission coefficient. Predicted:,$- d / h=0.324 ;---, d / h=0.570$; - -,$d / h=0.759$. Measured: $\quad, d / h=0.324 ; \boldsymbol{\Delta}, d / h=0.570$ $d / h=0.759$.

6. Same as Fig. 5 but for $r_{0}=0.6$.

7. Comparison of the predicted reflection and transmission coefficients with experimental results as a function of $k h$ for $d / h=0.378$ : (a) reflection coefficient, (b) transmission coefficient. Predicted:,$- r_{0}=0.3 ;---, r_{0}=0.4 ;-\cdots-$, $r_{0}=0.5 ;-\bullet-r_{0}=0.6$. Measured: $\bigcirc, r_{0}=0.3 ; \boldsymbol{\Delta}, r_{0}=0.4$; $r_{0}=0.5 ;+, r_{0}=0.6$.

8. Same as Fig. 7 but for $d / h=0.570$. 


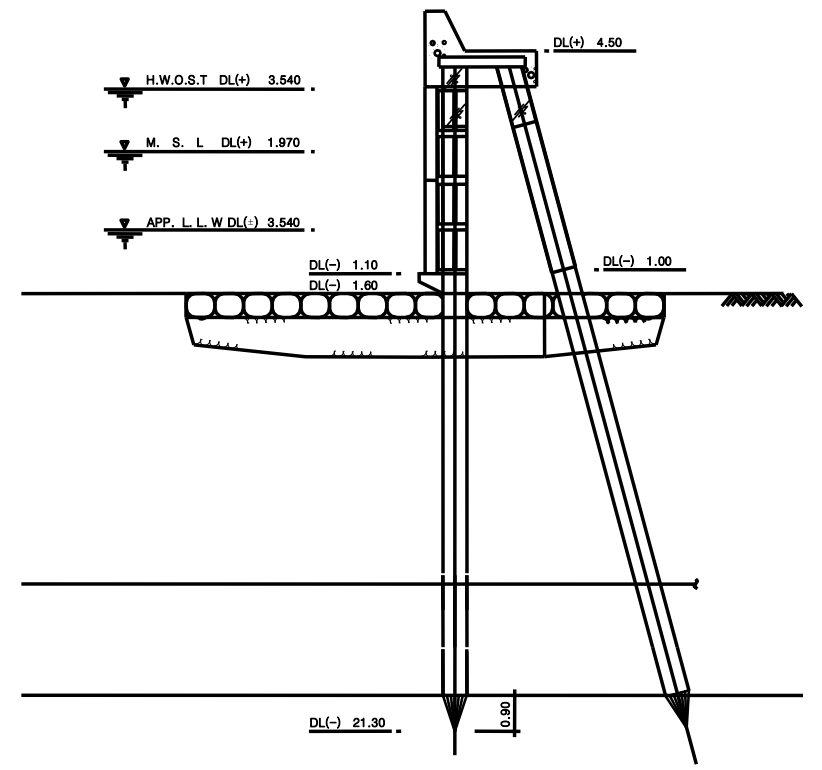

Fig. 1. Cross-section of the Yeoho Port breakwater. 


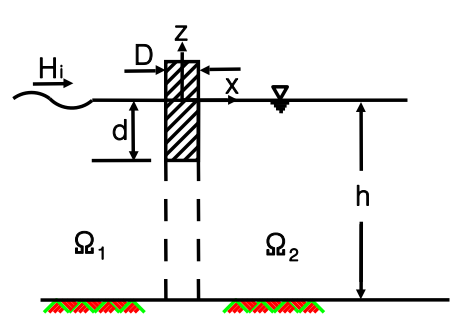

(a)

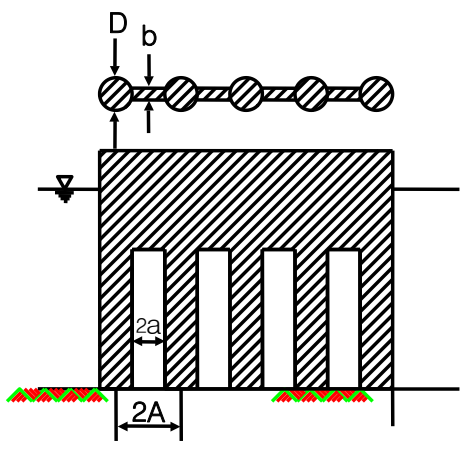

(b)

Fig. 2. Definition sketch: (a) side view, (b) front view and top view. 


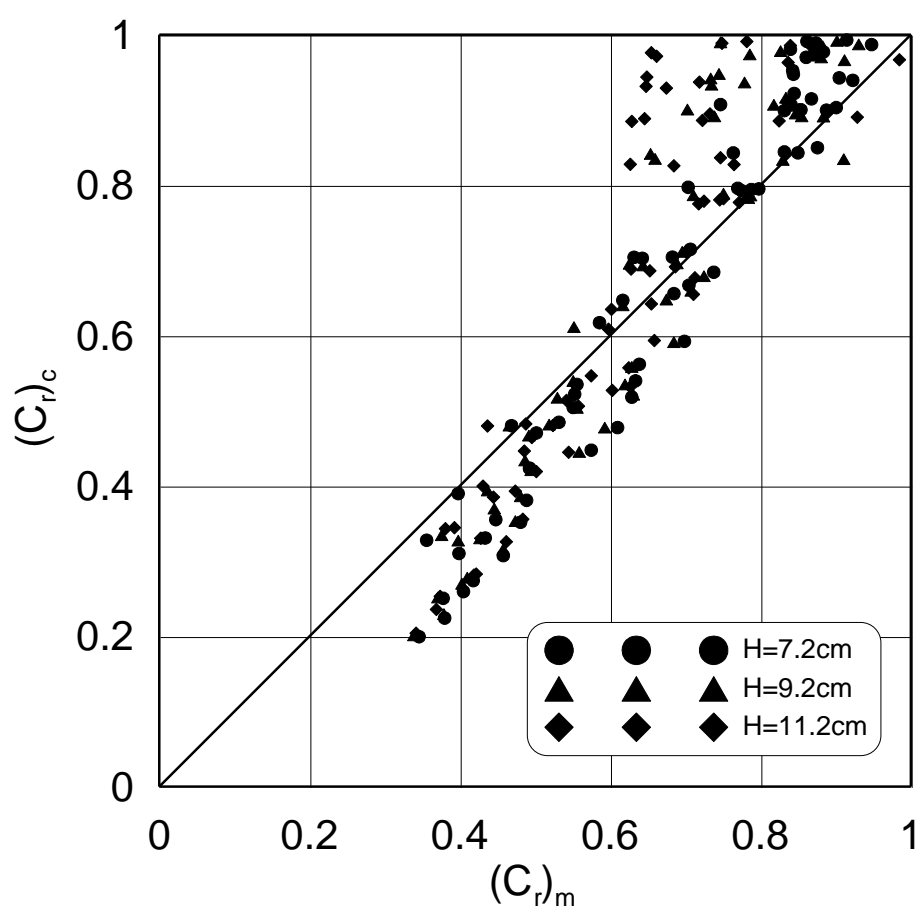

Fig. 3. Comparison of the reflection coefficients between measurement and calculation. 


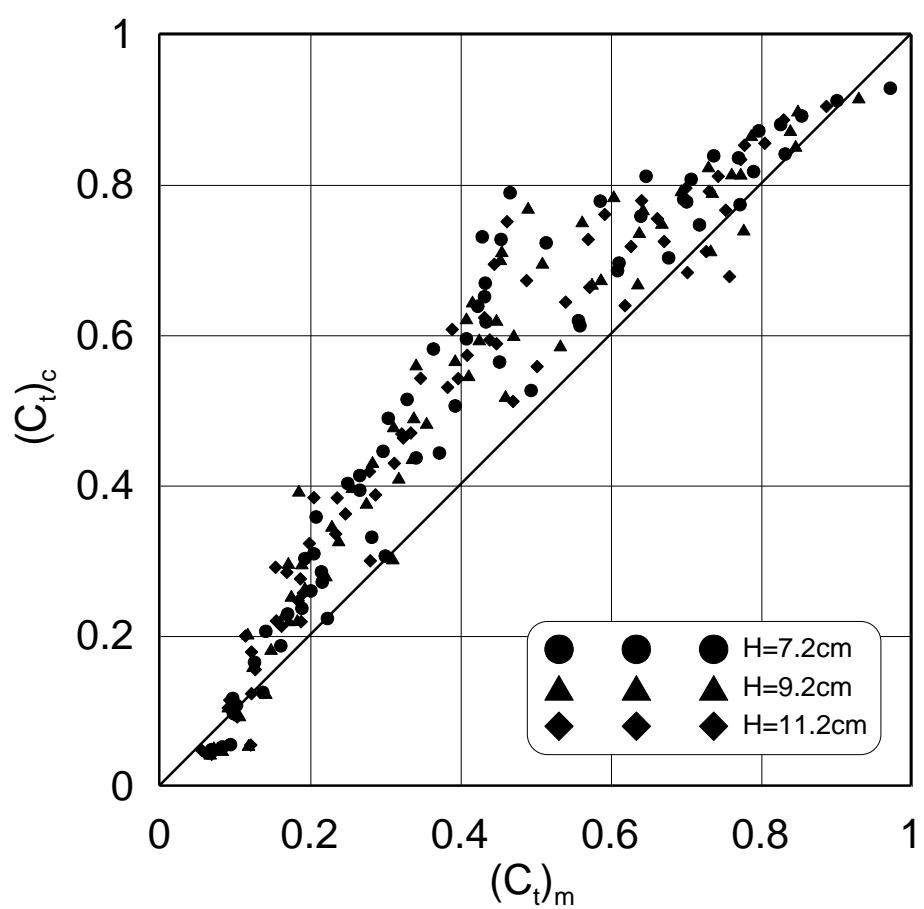

Fig. 4. Comparison of the transmission coefficients between measurement and calculation. 


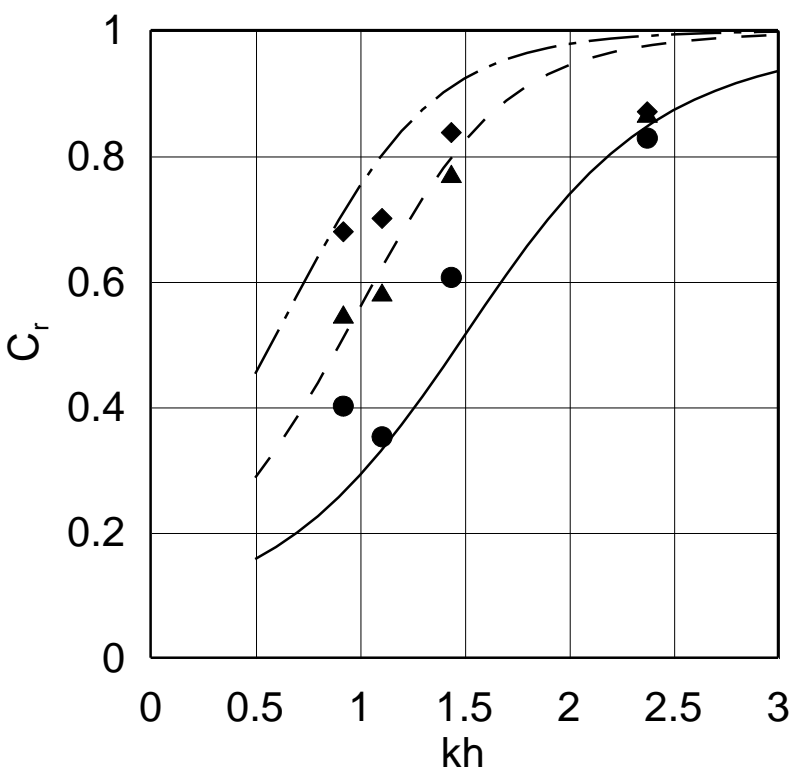

(a)

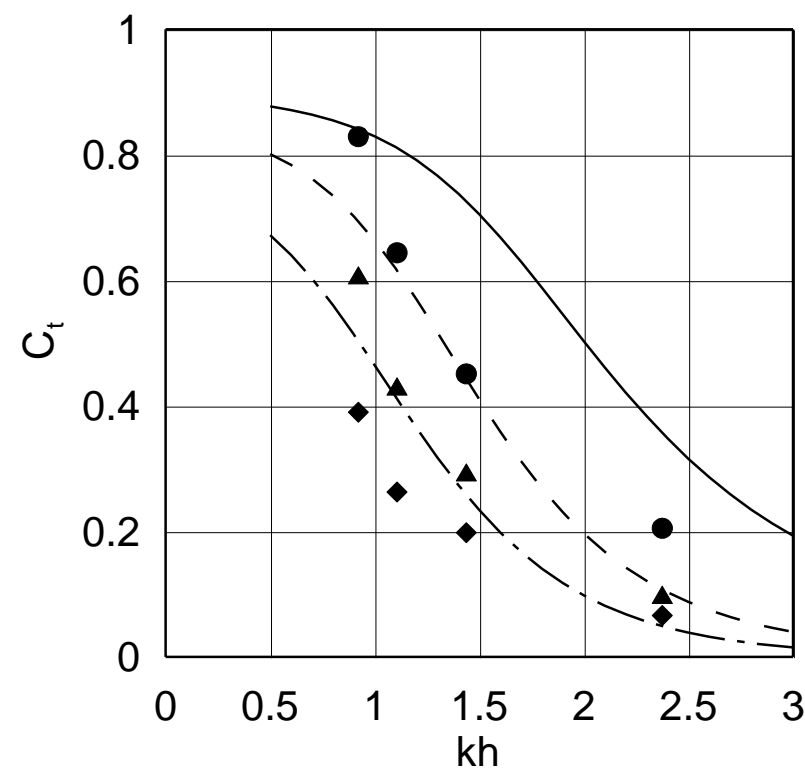

(b)

Fig. 5. Comparison of the predicted reflection and transmission coefficients with experimental results as a function of $k h$ for $r_{0}=0.4$ : (a) reflection coefficient, (b) transmission coefficient. Predicted: $\longrightarrow, d / h=0.324 ;--, d / h=0.570 ;-\cdots-$, $d / h=0.759$. Measured: $\bigcirc, d / h=0.324 ; \boldsymbol{\Delta}, d / h=0.570 ; \diamond, d / h=0.759$. 


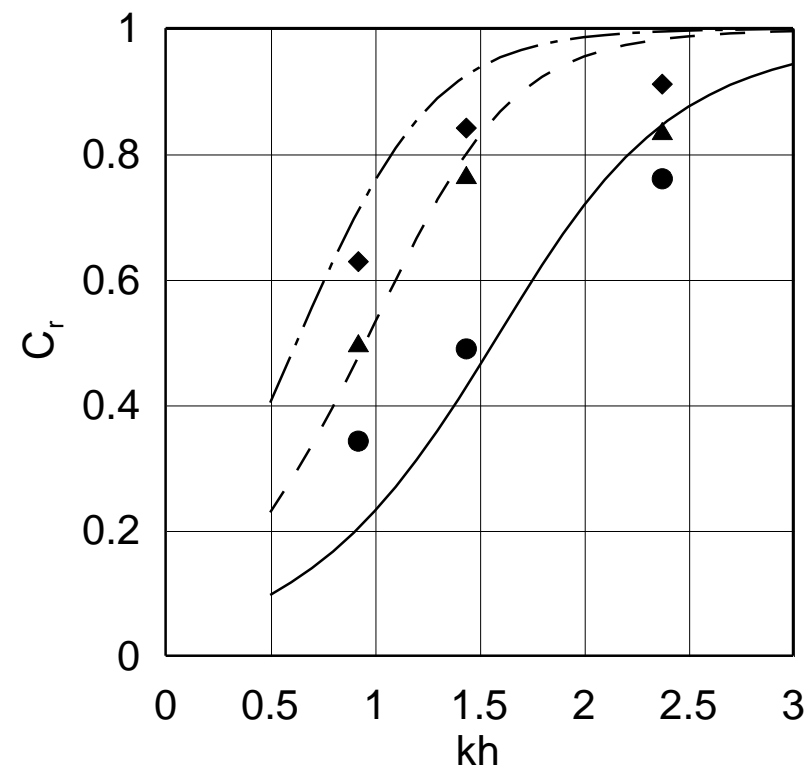

(a)

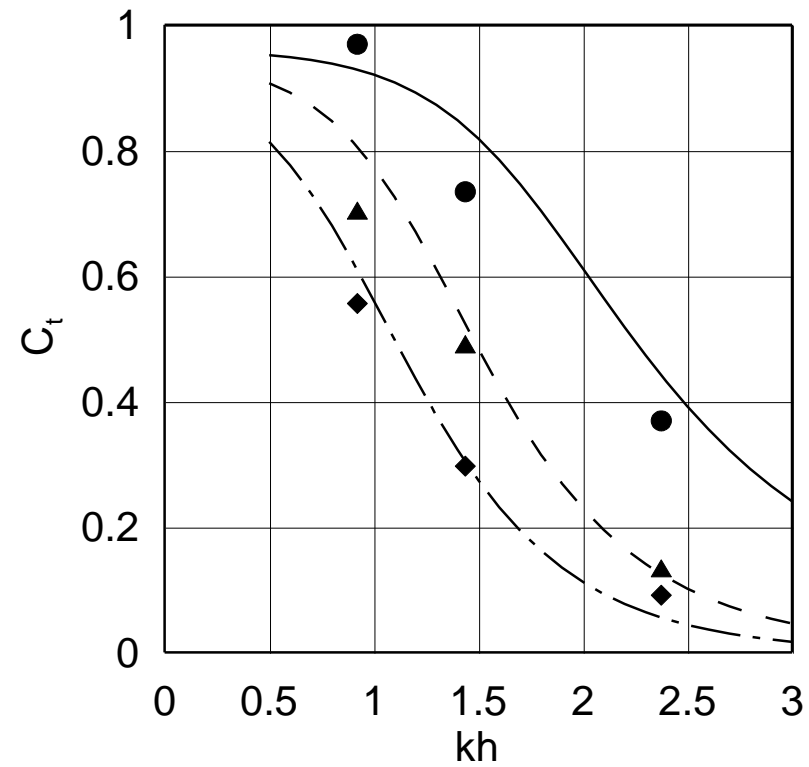

(b)

Fig. 6. Same as Fig. 5 but for $r_{0}=0.6$. 


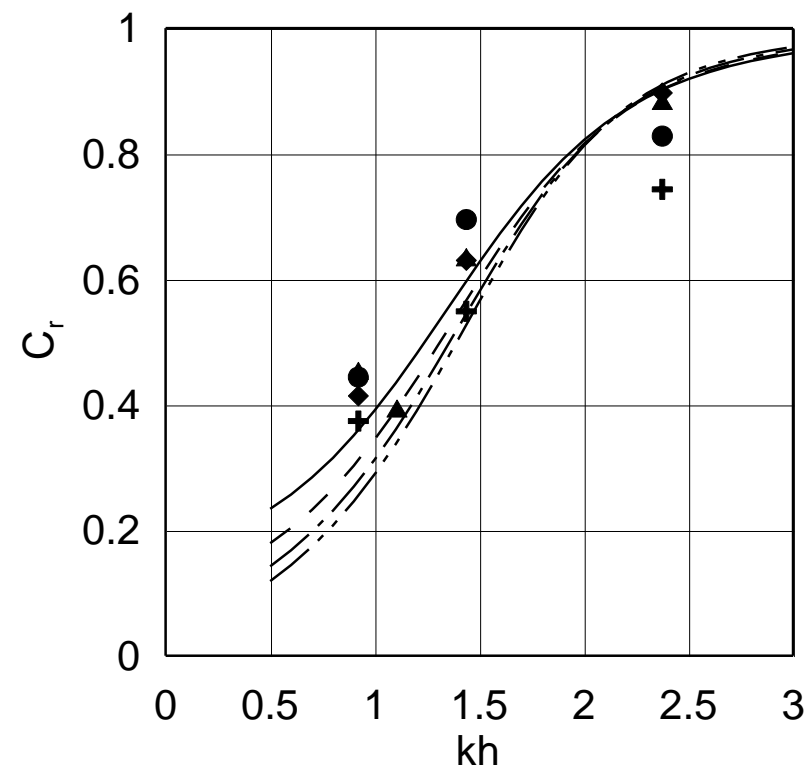

(a)

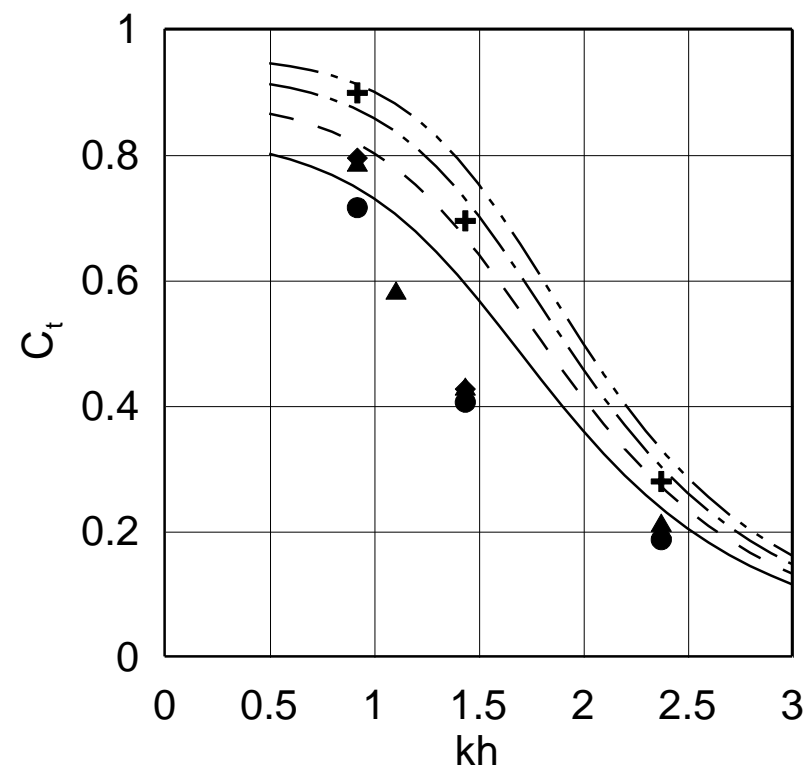

(b)

Fig. 7. Comparison of the predicted reflection and transmission coefficients with experimental results as a function of $k h$ for $d / h=0.378$ : (a) reflection coefficient, (b) transmission coefficient. Predicted:,$- r_{0}=0.3 ;--, r_{0}=0.4 ;-\cdots-, r_{0}=0.5$; - - 一 $r_{0}=0.6$. Measured: $\boldsymbol{○}, r_{0}=0.3 ; \boldsymbol{\Delta}, r_{0}=0.4 ; \diamond, r_{0}=0.5 ;+$, $r_{0}=0.6$. 


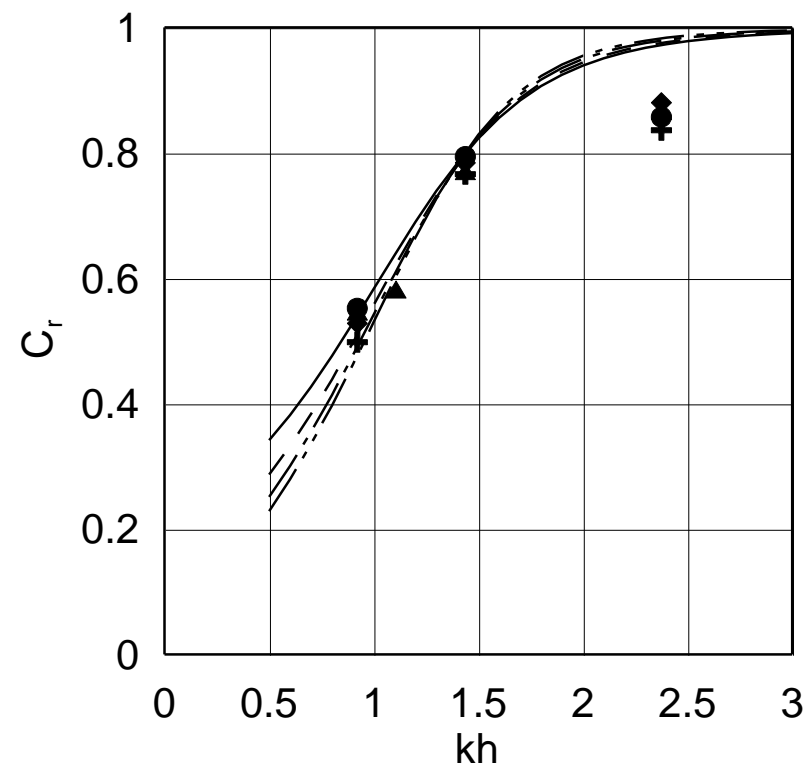

(a)

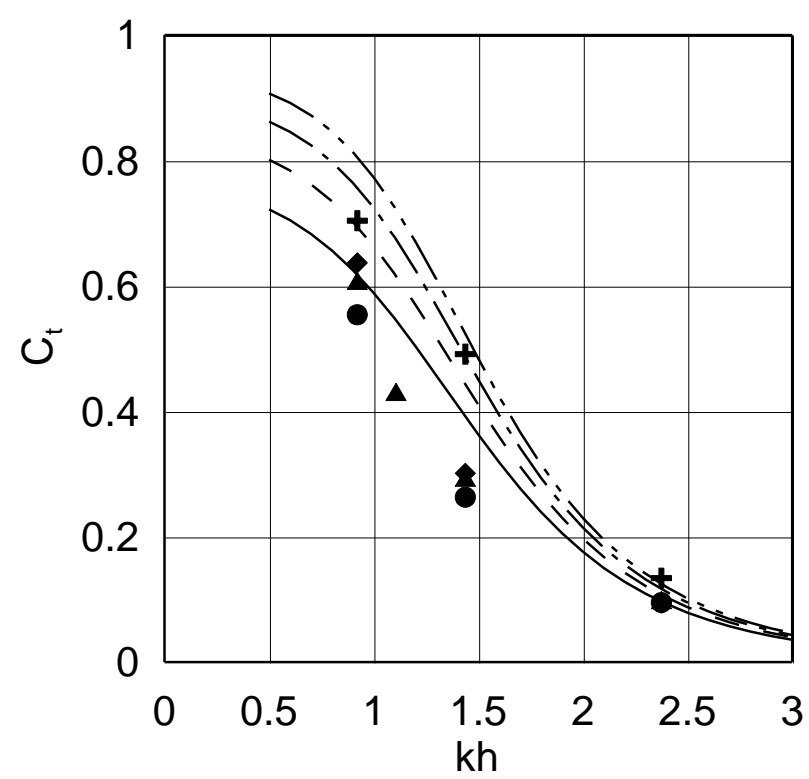

(b)

Fig. 8. Same as Fig. 7 but for $d / h=0.570$. 